\title{
CONOCIMIENTOS TRADICIONALES Y PROBLEMAS EN EL APROVECHAMIENTO DE RECURSOS PESQUEROS CON LOS COMERCIANTES DE VARIOS MERCADOS DE LA CIUDAD DE QUITO-ECUADOR
}

\author{
TRADITIONAL KNOWLEDGE AND PROBLEMS IN THE USE OF FISHING RESOURCES \\ WITH MERCHANTS OF SEVERAL MARKETS IN THE CITY OF QUITO-ECUADOR
}

\author{
Marco A. RODRÍGUEZ-SEGOVIA*, Fernanda PAZ-SUCONOTA, Anthony SORIANO, \\ María JUMBO
}

Universidad Central del Ecuador, Facultad de Ciencias Biológicas, Numa Pompillo Llona y Yaguachi, Quito- Ecuador. *Autor para correspondencia: marcozeus24@gmail.com

\begin{abstract}
RESUMEN
Los mercados municipales son una gran fuente de información para las investigaciones etnobiológicas, en ellos los pescadores satisfacen la constante demanda de determinadas especies de pescado. La etnoictiología estudia las relaciones que mantienen grupos humanos con los peces, se encarga de estudiar los conocimientos sobre los peces y su ambiente, así como las prácticas pesqueras para efectuar sus capturas. El presente estudio se enfocó en detectar e identificar algunos problemas relacionados al aprovechamiento de recursos pesqueros en varios mercados de Quito y presentar alternativas para solucionar esos problemas. Además de facilitar la divulgación y conservación del conocimiento tradicional sobre nombres comunes de muchas especies. Visitamos cinco mercados de la ciudad de Quito y pescaderías aledañas a estos mercados. En estos lugares realizamos entrevistas con preguntas abiertas a los comerciantes de pescado, el contenido de las preguntas tenía que ver con nombres comunes, especies más vendidas, escases de especies y manejo de peces que no logran venderse. Entrevistamos a 35 vendedores. Algunas de las 43 especies encontradas poseían más de un nombre común. Entre las especies más vendidas destacaron Peprilus medius (11\%), Sphyraena ensis (10\%), Hemanthias signifer (10\%), Bagre pinnimaculatus (9\%), Scomberomorus sierra (7\%) y Thunnus albacares (7\%). Existieron unos pocos ejemplares pequeños e inmaduros entre los peces observados. Solo $23 \%$ de las personas mencionaron escases de las especies: Lutjanus argentiventris, Centropomus robalito, Sphyraena ensis, y posiblemente de Cynoscion sp. y Larimus sp. El $40 \%$ de los vendedores vendían todo su pescado, una pequeña parte lo congelaba, transformaba el pescado en harina o también los regalaban. Solo el 3\% de los entrevistados los desechaba cuando no lograba venderlos. En conclusión, los problemas identificados tenían algunas alternativas de solución que al ser revisadas parecen muy viables. Además, se logró recopilar una gran riqueza de nombres comunes.
\end{abstract}

Submitted: 16/05/2020; Accepted: 21/06/2020

Palabras-clave: Consumo; Descarte; Escases; Nombres comunes; Sobrepesca

\begin{abstract}
Municipal markets are a great source of information for ethnobiological research, in which fishermen satisfy the constant demand for certain species of fish. Ethnoictiology studies the relationships between human groups and fish, is responsible for studying knowledge about fish and its environment, as well as fishing practices to catch them. The present study focused on detect and identify some problems related to the use of fishery resources in various markets in Quito and presenting alternatives to solve these problems. In addition to facilitating the dissemination and conservation of traditional knowledge about common names of many fish species. For this, we visited five markets in the city of Quito and nearby fishmongers from these markets. In these places we conducted interviews with open questions to fish sellers, the content of the questions had to do with the common names, best-selling species, scarcity of species and handling of fish that they can't sell. We interviewed 35 sellers . Some of the 43 species that we found had more than one common name. Among the best-selling species there were Peprilus medius (11\%), Sphyraena ensis (10\%), Hemanthias signifer (10\%), Bagre pinnimaculatus (9\%), Scomberomorus sierra (7\%) and Thunnus albacares (7\%). A few small, immature specimens existed among the observed fish. Only $23 \%$ of people mentioned shortages of fish species, these were Lutjanus argentiventris, Centropomus robalito, Sphyraena ensis and possibily Cynoscion sp. and Larimus sp. $40 \%$ of the vendors sold all their fish, a small part froze it, transformed the fish into flour or gave it away as well. Only $3 \%$ of the respondents discarded them when he could not sell them. In conclusion, the identified problems had some alternative solutions that, when reviewed, seem very viable. In addition, it was possible to compile a great wealth of common names
\end{abstract}

Keywords: Consumption; Discard; Scarcity; Common names; Overfishing 


\section{INTRODUCCIÓN}

En Ecuador la mayor parte de los productos pesqueros obtenidos del mar se distribuyen con prioridad en la Región Costa, la demanda se concentra en ciudades-puertos, puntos de desembarque, zonas costeras y lugares aledaños (PONTÓN, 2015). El resto se envían mayormente a la región Sierra donde el porcentaje de consumo es más reducido debido a la distancia geográfica, abastecimiento y cultura gastronómica (PONTÓN, 2015).

En la pesca industrial la venta es directa hacia procesadores industriales o a mayoristas mientras que la pesca artesanal dirige sus ventas hacia un gran número de intermediarios (PONTÓN, 2015). Los intermediarios llevan muchos de los productos del mar hacia los mercados de Quito donde se venden a los consumidores. También hay pescadores independientes que traen una gran variedad de especies de diferentes provincias y hábitats; de manera que satisfacen constantemente la demanda hacia los mercados (FAO, 2016). En los mercados de la ciudad de Quito, los comerciantes de pescado son abastecidos tanto por intermediarios como por pescadores. De hecho, algunos de estos comerciantes también se dedican a ser pescadores como parte de sus actividades de trabajo.

En la ciudad de Quito los mercados y ferias públicos son de vital importancia para el abastecimiento de alimentos, este sistema abastece a cerca del 75\% de la población (HOLLENSTEIN, 2019). Los Mercados de Quito tienen la capacidad de garantizar la soberanía alimentaria, la soberanía económica, el tejido social del sector urbano y relaciones equitativas con las regiones que abastecen alimentos (HOLLENSTEIN, 2019). Tienen un alto valor cultural debido a la antigüedad de algunos de ellos y su uso para los habitantes (HOLLENSTEIN, 2019). Los mercados tradicionales son una fuente inexplorada para las investigaciones etnobiológicas, una gran información sobre la biodiversidad, manejo y aspectos culturales de especies se puede obtener de estos lugares (MAIOLI-AZEVEDO Y DA FONSECA-KRUEL, 2007).

Los comerciantes de pescado en los mercados son actores fundamentales para entender algunas dinámicas en el aprovechamiento de los recursos pesqueros y pueden ser de gran ayuda en la resolución de problemas relacionados a estos recursos. Conocer de primera mano las percepciones de los comerciantes ha sido útil para identificar problemas relacionados con temas de sobrepesca, especies amenazadas, demanda excesiva, escases de especies y desperdicio de alimentos. Indicios como la presencia de especies amenazadas comercializadas en mercados, pueden ser útiles para despertar la atención de autoridades y generar indagaciones sobre el impacto en sus formas de manejo y extracción (MAIOLI-AZEVEDO Y DA FONSECA-KRUEL, 2007). Frente a los problemas citados existen alternativas con soluciones viables.

Actualmente vivimos en una época donde la disponibilidad de los recursos pesqueros está comprometido debido principalmente a la sobrepesca (FAO, 2016). Ecuador es un país que pesca y exporta grandes cantidades de pescado; de acuerdo a Ron (2012), en el país el consumo promedio anual de kilo de pescado por persona llegaba a bordear entre 5 a $8 \mathrm{~kg}$, en comparación al consumo mundial de 12 a $16 \mathrm{~kg}$. En 2014 el consumo mundial aumento alcanzando los $20 \mathrm{~kg}$ por persona (FAO, 2016).

La creciente sobrepesca genera reducción de la talla de los peces, menos capturas, escases de los recursos hidrobiológicos y pone en peligro de extinción a las especies (GUEVARA-CARRASCO, 2004; AUNAP y UNIMAGDALENA, 2013). La tendencia mundial al respecto es observar un incremento en el número de pesquerías insostenibles (FINKBEINER et al., 2017). Esta problemática es grave porque genera pérdida de la biodiversidad y alteraciones en la estructura y función de los ecosistemas; también puede afectar la manera en la que se generan beneficios socioeconómicos de las actividades pesqueras (COLL et al., 2008; FINKBEINER et al., 2017).

La sobrepesca ha ocurrido tan solo en una pequeña fracción de especies como resultado de la pesca intensiva y selectiva (ZHOU et al., 2015).Muchas especies capturadas en grandes volúmenes son víctimas de sobreexplotación generada por una alta demanda (CINNER y MCCLANAHAN, 2006; ZHOU et al., 2015), lo que al ser analizado puede revelar como trasfondo a la falta de medidas en el adecuado aprovechamiento sostenible de los recursos pesqueros (KELLEHER, 2008; BOON et al., 2011). En el caso de los mercados de Quito la demanda alta de varias especies, escases de algunas y presencia de peces pequeños son factores que podrían señalar síntomas de sobrepesca (AUNAP y UNIMAGDALENA, 2013)

Sumado a la sobrepesca el descarte de las capturas y paulatino deterioro de los peces durante su distribución generan un enorme desperdicio de alimentos y evidencian un gran derroche económico de los recursos pesqueros a nivel mundial (FAO, 2012). Un estudio realizado por la FAO en 2005 dice 
que el descarte mundial de la pesca fue de 7,3 millones de toneladas (FAO, 2012). En la actividad pesquera la pérdida de alimentos ocurre en las etapas de producción, postcosecha, y procesamiento de la cadena de abastecimiento de los peces; las pérdidas ocurren al final de la cadena y está más relacionado al comportamiento de los vendedores minoristas y del consumidor (PARFITT et al., 2010; FAO, 2012). En los mercados de Quito el desperdicio de alimentos es algo cotidiano, inclusive para los comerciantes de pescado. Los comerciantes de pescado han implementado algunas alternativas plausibles para evitar en lo posible arrojar pescado a la basura cuando no se logran vender.

Aparte de los problemas mencionados anteriormente, los comerciantes de pescado en los mercados de Quito poseen conocimientos tradicionales interesantes para la Etnobiología. Una rama de la Etnobiología es la Etnozoología, la cual busca comprender las interrelaciones de grupos humanos con la fauna silvestre de su entorno (FARIÑA et al., 2011). Dentro de esta, la Etnoictiología abarca el estudio de las relaciones que mantienen grupos humanos con los peces, destacando sus percepciones, formas de clasificar a los animales de acuerdo con sus usos (alimenticios, medicinales, carnada, económicos, actividades rituales, etc.) y taxonomía popular (FARIÑA et al., 2011). También se encarga de estudiar los conocimientos sobre los peces y su ambiente, así como las prácticas pesqueras para efectuar sus capturas (FARIÑA et al., 2011; MARTINO, 2016).

Algunas publicaciones etnoictiológicas han destacado el uso de nombres comunes o nativos en compañía de nombres científicos con el propósito de hacer posible el acceso al conocimiento general y facilitar su difusión; de manera que sean preservados y ayuden en la identificación taxonómica para las personas que se dedican a la investigación etnobiológica (ANGULO, 2013). El estudio de nombres comunes es importante en perspectiva a la pérdida de conocimientos ocasionada por el deficiente relevo generacional de pescadores y comerciantes de pescado (NASS et al., 2010). Esto debido al poco incentivo socioeconómico y cultural de las actividades pesqueras; por lo que muchos evitan trabajar en ellas (NASS et al., 2010). Esta pérdida responde a procesos de transculturalización, que hacen que las personas o grupos humanos adquieran elementos culturales prestados de otra cultura (FARIÑA et al., 2011). Con el tiempo estos procesos terminan por generar aculturación, un estado en el que básicamente una cultura ha incorporado elementos de una cultura ajena, sustituyendo su cultura original. (GARCÍA Y FIGUEROA, 2007; FARIÑA et al., 2011; ZEBADÚA, 2011).

El presente estudio se enfocó en detectar e identificar algunos problemas relacionados al aprovechamiento de recursos pesqueros en varios mercados de Quito y revisar alternativas presentadas para solucionar esos problemas. Además de facilitar la divulgación y conservación del conocimiento tradicional sobre nombres comunes de muchas especies de peces.

\section{MATERIAL Y MÉTODOS \\ Área de estudio}

El presente estudio se realizó en Ecuador, provincia de Pichincha, en cinco mercados y varias pescaderías aledañas a los alrededores de dichos mercados dentro del Distrito Metropolitano de Quito. En Quito existen unos 42 mercados y ferias libres temporales (HOLLENSTEIN, 2019). Los mercados en este trabajo fueron seleccionados de manera preferencial por ser de los más concurridos en Quito. Los mercados estudiados fueron: el Mercado Mayorista, el Mercado Chiriyacu el Mercado de San Roque, el Mercado de Cotocollao y el Mercado de la Ofelia (ver Tabla 1 y Figura 1).

\section{Metodología}

En el presente estudio se realizaron 35 entrevistas con preguntas abiertas (en trabajos etnobiológicos se recomiendan una muestra de al menos 35 entrevistas o superior para un esfuerzo de muestreo adecuado (HOFFMAN y GALLAHER, 2007; RODRÍGUEZ et al., 2018). Las entrevistas estaban dirigidas a cualquiera de todos los posibles comerciantes de pescado que trabajan en los mercados y pescaderías aledañas mencionados anteriormente (algunos de los comerciantes también se dedicaban a ser pescadores). La edad de los entrevistados iba desde los 24 a 70 años, con un promedio de edad de 37 años. Se entrevistaron a 27 varones y 8 mujeres. Para recopilar la información, se realizaron visitas a los puestos de venta de pescado entre los meses de noviembre de 2019 a febrero del 2020 (Figura 2). Todos los participantes fueron informados sobre los objetivos del trabajo, obteniendo su consentimiento hablado para participar. 
Tabla. 1. Mercados visitados en la ciudad de Quito.

\begin{tabular}{ll}
\hline Mercados & Coordenadas UTM \\
\hline Mercado Mayorista & $774429.810 \mathrm{E} 9969966.032 \mathrm{~N}$ \\
Mercado de San Roque & $775878.177 \mathrm{E}$ 9975657.704N \\
Mercado de Cotocollao y & $779334.815 \mathrm{E}$ 9987168.261N \\
sus alrededores & \\
Mercado Chiriyacu & $776160.179 \mathrm{E}$ 9972333.330N \\
Mercado de la Ofelia & $779299.273 \mathrm{E} 9987550.559 \mathrm{~N}$ \\
\hline
\end{tabular}
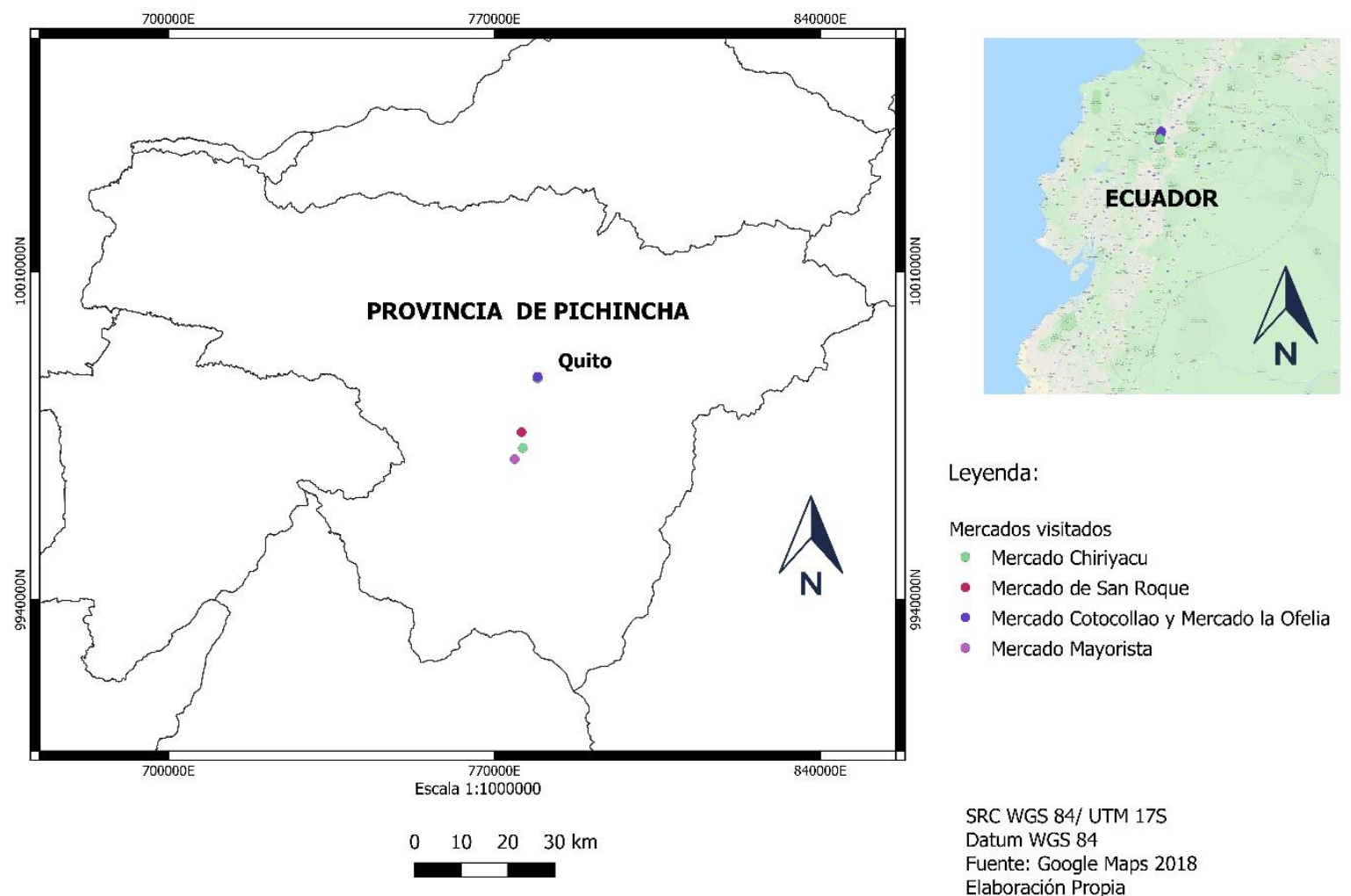

Leyenda:

Mercados visitados

Mercado Chiriyacu

- Mercado de San Roque

- Mercado Cotocollao y Mercado la Ofelia

- Mercado Mayorista

Figura 1: Localización de los Mercados visitados en la Provincia de Pichincha.

Las preguntas incluidas en las entrevistas tuvieron que ver con nombres comunes, demanda de peces, escases de peces y desperdicio de peces (Anexo I). También nos ayudamos de la observación directa para tomar nota de estos aspectos. También se contó con la ayuda de una cámara fotográfica para registrar las especies de peces citadas y aquellas que no pudimos identificar. Para la fotoidentificación de estas últimas, utilizamos la guía de peces marinos del Ecuador Continental (Tomo II) de Jiménez-Prado y Beárez (2004) y la guía de peces para aguas continentales en la vertiente occidental del Ecuador de Jiménez-Prado et al. (2015). En algunas ocasiones y por mera casualidad detectamos la presencia de ejemplares juveniles de algunas especies, tomamos en cuenta su presencia en los puestos de venta de varios entrevistados. Adicionalmente revisamos si entre las especies, alguna de ellas estaba en alguna categoría de amenaza al buscarlas en la lista roja de especies amenazadas de la UICN (2020). Los datos obtenidos fueron organizados en una base de datos para determinar la frecuencia de ocurrencia de cada especie o respuesta (número de citaciones provistas para cada especie o respuesta dividida para el número de informantes por cien). 


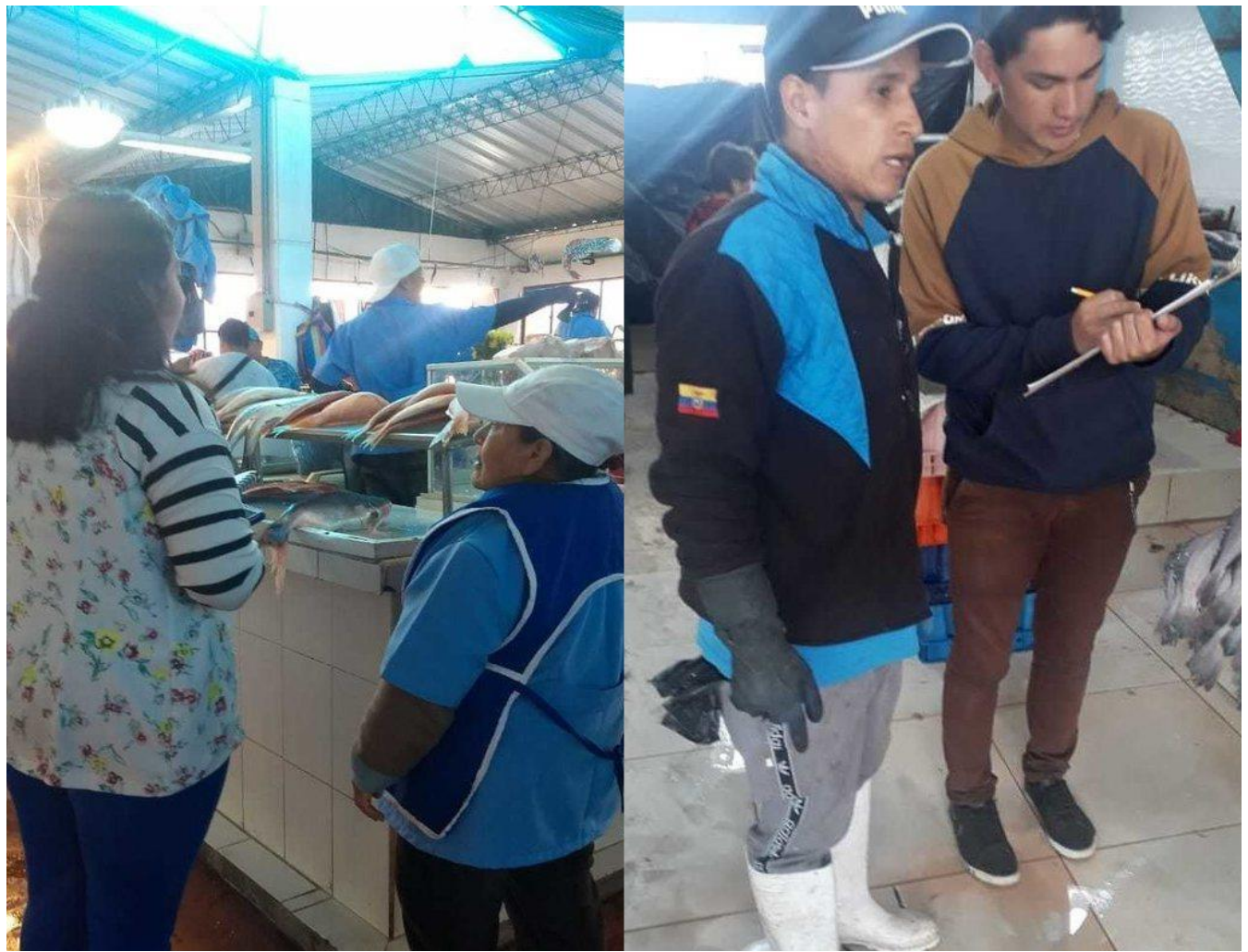

Figura 2. Entrevistas realizadas con el consentimiento previo informado a los vendedores de pescado en mercados de Quito. Todos los participantes fueron informados sobre el objetivo del trabajo.

\section{RESULTADOS Y DISCUSIÓN}

Nombres comunes usados por los vendedores de pescado

Entre las 35 entrevistas realizadas en los mercados visitados se registraron 43 especies de peces, 36 eran especies marinas y 7 eran de agua dulce. Sobre las especies de agua dulce, 5 son especies nativas usadas para el consumo, estas especies eran: Transancistrus aequinoctialis, Hoplias malabaricus, Adinoacara rivulatus, Dormitator latifrons y Piaractus brachypomus. El resto de peces de agua dulce eran Oreochromis niloticus y Oreochromis aureus, que son especies introducidas. De varias especies registramos más de un nombre común, de los cuales algunos eran más usados que otros (Tabla 2 y Anexo II). Los nombres comunes con los que los vendedores conocen a una especie pueden ser similares, tal es el caso de Peprilus medius cuyo nombre común varía entre Pampanito, Pampano, Carita y Mampanito. En otras ocasiones los nombres comunes designan a más de una especie o inclusive difieren bastante para una misma especie. Curiosamente la mayoría de los peces de color rojo son llamados Pargos a excepción de O. aureus. Algunas especies solo pudieron ser identificadas hasta el nivel taxonómico de género debido a que son procesadas de forma rápida en filetes para la venta como el caso de algunas corvinas (Cynoscion spp.), de manera que nos basamos en la observación de los restos de los animales.

Las especies de peces de agua dulce nativas fueron muy escasas entre los Mercados visitados. Son peces que normalmente se usan para la pesca de subsistencia, pertenecen a tierras bajas de las regiones Costa y Amazonía del Ecuador, como el caso de H. malabaricus; en ocasiones llegan hasta los mercados de Quito y no poseen un comercio muy desarrollado (JIMÉNEZ-PRADO et al., 2015). T. aequinoctialis tiene un uso histórico bien documentado. Jiménez-Prado et al. (2015) menciona que dicha especie, así como todas las especies afines a su género y a la familia Loricariidae son muy utilizadas y apreciadas en la alimentación, siendo una delicia en la preparación de caldos. Comúnmente este tipo de peces se conocen también como "Preñadillas" y han sido pescados por indígenas desde tiempos precolombinos (BURGOS et al., 2012). Jiménez-Prado et al. (2015) presenta a A. rivulatus como una especie de vital importancia alimenticia para los pescadores de Vinces (Provincia de los Rios-Región Costa), en esta ocasión reportamos su venta en la ciudad de Quito. $D$. latifrons y P. brachypomus son especies que suelen cultivarse en la piscicultura ecuatoriana (FAO, 2010; JIMÉNEZ-PRADO et al., 2015). 
Tabla 2. Nombres comunes para varias especies de peces en mercados de Quito. Los nombres comunes son mostrados en orden descendente acorde a la frecuencia de ocurrencia con el que fueron citados.

\begin{tabular}{|c|c|c|c|}
\hline$\#$ & Familia & Nombre científico & Nombres comunes \\
\hline 1 & Ariidae & Bagre pinnimaculatus & Bagre $(60 \%)$ \\
\hline 2 & Ariidae & Occidentarius platypogon & Bagre $(6 \%)$ \\
\hline 3 & Carangidae & Selene peruviana & Carita $(17 \%) /$ Cara $(3 \%)$ \\
\hline 4 & Carangidae & Trachinotus paitensis & Verugata $(3 \%)$ \\
\hline 5 & Carangidae & Caranx caballus & Jurel (3\%) \\
\hline 6 & Carangidae & Selene oerstedii & Carita $(26 \%)$ \\
\hline 7 & Carangidae & Oligoplites altus & Voladora $(3 \%)$ \\
\hline 8 & Centropomidae & Centropomus robalito & Róbalo (14\%) \\
\hline 9 & Cichlidae & Oreochromis niloticus & Tilapia (34\%)/Tilapia negra (6\%) \\
\hline 10 & Cichlidae & Oreochromis aureus & Tilapia roja (11\%)/Tilapia (3\%) \\
\hline 11 & Cichlidae & Andinoacara rivulatus & Vieja de agua dulce (3\%) \\
\hline 12 & Coryphaenidae & Coryphaena hippurus & Dorado $(6 \%)$ \\
\hline 13 & Eleotridae & Dormitator latifrons & Chame (3\%) \\
\hline 14 & Erythinidae & Hoplias malabaricus & Guanchiche (3\%) \\
\hline 15 & Gerreidae & Diapterus peruvianus & Mojarra (29\%)/Roncador (9\%) \\
\hline 16 & Haemulidae & Haemulopsis axillaris & Pargo blanco (3\%) \\
\hline 17 & Haemulidae & Haemulon flaviguttatun & Sol $(3 \%)$ \\
\hline 18 & Haemulidae & Anisotremus interruptus & Roncador $(3 \%)$ \\
\hline 19 & Haemulidae & Pomadasys macracanthus & Pargo blanco (3\%) \\
\hline 20 & Haemulidae & Orthopristis chalceus & Chullo $(3 \%)$ \\
\hline 21 & Loricariidae & Transancistrus aequinoctialis & Campeche $(3 \%)$ \\
\hline 22 & Lutjanidae & Lutjanus colorado & Pargo $(3 \%)$ \\
\hline 23 & Lutjanidae & Lutjanus guttatus & Pargo lunarejo (3\%) \\
\hline 24 & Lutjanidae & Lutjanus argentiventris & Pargo $(14 \%)$ \\
\hline 25 & Malacanthidae & Caulolatilus affinis & Cabezudo (3\%) \\
\hline 26 & Mugilidae & Mugil cephalus & Lisa $(20 \%)$ \\
\hline 27 & Mullidae & Mulloidichthys dentatus & Chivo $(3 \%)$ \\
\hline 28 & Paralichthyidae & Syacium latifrons & Lenguado (3\%) \\
\hline 29 & Sciaenidae & Cynoscion sp. & Corvina $(34 \%)$ \\
\hline 30 & Sciaenidae & Cynoscion phoxocephalus & Corvina chateada $(3 \%)$ \\
\hline 31 & Sciaenidae & Umbrina xanti & Rabo amarillo (3\%) \\
\hline 32 & Scombridae & Thunnus obesus & Albacora $(6 \%)$ \\
\hline 33 & Scombridae & Euthynus lineatus & Atún $(20 \%) /$ Albacora $(20 \%)$ \\
\hline 34 & Scombridae & Katsuwonus pelamis & Albacora (6\%)/Atún (3\%)/ \\
\hline 35 & Scombridae & Thunnus albacares & Atún (3\%)/Albacora (3\%) \\
\hline 36 & Scombridae & Scomberomorus sierra & Sierra $(54 \%)$ \\
\hline 37 & Scorpaenidae & Pontinus sierra & Pargo rojo (3\%) \\
\hline 38 & Serranidae & Hemanthias signifer & $\begin{array}{l}\text { Pargo }(51 \%) / \text { Pargo rojo }(26 \%) / \text { Gringo }(3 \%) / \\
\text { Rojo }(3 \%)\end{array}$ \\
\hline 39 & Serranidae & Diplectrum eumelum & Camotillo (3\%) \\
\hline 40 & Serranidae & Hemanthias peruanus & Pargo (3\%) \\
\hline 41 & Serrasalmidae & Piaractus brachypomus & Cachama (3\%) \\
\hline 42 & Sphyraenidae & Sphyraena ensis & Picudo/a (51\%) \\
\hline & Stromateidae & Peprilus medius & $\begin{array}{l}\text { Pampanito (40\%)/Pampano (11\%)/ Carita (9\%)/ } \\
\text { Mampanito (3\%) }\end{array}$ \\
\hline
\end{tabular}




\section{Peces con mayor demanda para el consumo}

Las especies de peces más vendidas citadas durante las entrevistas tomadas a vendedores en Mercados de Quito fueron: Peprilus medius (11\%), Sphyraena ensis (10\%), Hemanthias signifer (10\%) Bagre pinnimaculatus (9\%) Scomberomorus sierra (7\%) y Thunnus albacares (7\%) (Tabla 3).

Tabla 3. Peces más vendidos en mercados del Distrito Metropolitano de Quito.

\begin{tabular}{lc}
\hline Especies citadas & Frecuencia de ocurrencia \\
\hline Peprilus medius & $11 \%$ \\
Sphyraena ensis & $10 \%$ \\
Hemanthias signifer & $10 \%$ \\
Bagre pinnimaculatus & $9 \%$ \\
Scomberomorus sierra & $7 \%$ \\
Thunnus albacares & $7 \%$ \\
Oreochromis niloticus & $6 \%$ \\
Selene peruviana & $5 \%$ \\
Cynoscion sp. & $5 \%$ \\
Centropomus robalito & $4 \%$ \\
Oreochromis aureus & $3 \%$ \\
Katsuwonus pelamis & $3 \%$ \\
Lutjanus argentiventris & $3 \%$ \\
Selene oerstedii & $3 \%$ \\
Pomadasys macracanthus & $3 \%$ \\
Euthynnus lineatus & $2 \%$ \\
Coriffaena hippurus & $2 \%$ \\
Lutjanus colorado & $1 \%$ \\
Haemulopsis axillaris & $1 \%$ \\
Lutjanus guttatus & $1 \%$ \\
Pontinus sierra & $1 \%$ \\
Diplectrum eumelum & $1 \%$ \\
Thunnus obesus & $1 \%$ \\
\hline
\end{tabular}

De acuerdo a la FAO (2020), muchas de las especies citadas entre las más vendidas son de gran importancia económica, especialmente aquellas especies con una industria muy desarrollada en Ecuador. Así la industria atunera en el país se basa principalmente en la pesca de T. albacares además de $T$. obesus, $K$. pelamis y E. lineatus $(\mathrm{FAO}, 2020)$. La popularidad en la alta demanda de varias especies de atún es un caso ejemplar respecto del agotamiento de los recursos pesqueros, pues el $30 \%$ de las poblaciones de atún están sobreexplotadas o agotadas y el 53\% están totalmente explotadas (BOON et al., 2011).

En general no encontramos especies amenazadas salvo por la excepción de T. obesus que es considerada una especie vulnerable (VU) y $T$. albacares que se considera casi amenazada (NT) y con sus poblaciones en detrimento de acuerdo a la UICN (2020). Recientemente las pesquerías han optado por pescar más especies pequeñas de niveles tróficos inferiores, debido a la reducción en las poblaciones de peces grandes que por lo general son depredadores (MOLFESE et al., 2014). Los atunes, por ejemplo, son depredadores. Así, cuando las pesquerías eliminan primero a los grandes depredadores, sus presas proliferan afectando la productividad y estabilidad del ecosistema (MOLFESE et al., 2014).

Especies más pequeñas citadas por nuestros informantes como S. sierra, H. signifer, B. pinnimaculatus, entre otros, son de mayor relevancia para la pesca blanca (FAO 2020). P. medius y $S$. ensis suelen tener un gran volumen de captura y esta es otra razón por las que son muy frecuentes en los mercados visitados, además de que son apreciadas por su sabor (CUCALÓN, 2015; ZABALALEAL et al., 2018). Las tilapias son peces introducidos con un amplio cultivo en el Ecuador.

La sobrepesca de especies se puede reducir desplazando el esfuerzo de pesca hacia poblaciones de especies de peces subutilizadas (ZHOU et al., 2015). También la implementación de cuotas de pesca y capturas compartidas entre pesquerías puede ayudar a balancear la cantidad de peces 
capturados y evitar problemas de pérdida de biodiversidad junto al subsecuente descarte y desperdicio (COSTELLO et al., 2008; KELLEHER, 2008; BOON et al., 2011).

\section{Especies que escasean}

De las entrevistas realizadas en solo un 23\% (8) de ellas obtuvimos información sobre especies de peces que están escaseando. Estos fueron: Lutjanus argentiventris, Centropomus robalito, Sphyraena ensis, la corvina Rosada y la Nata (estas dos últimas especies solo fueron mencionadas, pero no observadas para una buena identificación taxonómica, aunque posiblemente pudo tratarse de Cynoscion sp. y Larimus sp. respectivamente). Ninguna de ellas se encuentra en una categoría de amenaza (UICN 2020). Las razones explicadas por los comerciantes varían: 17\% (6) de ellos mencionaron que a veces no les llegan suficientes de estos peces por parte de sus proveedores y desconocen las razones; mientras que solo $6 \%$ (2) lo atribuyeron como causa a la demanda excesiva, lo que genera sobrepesca y conllevaría a la extinción de tales especies.

C. robalito y $S$. ensis fueron mencionados en problemas de escasez. De acuerdo a la UICN (2020), aunque son especies muy apreciadas en la pesca, su estado de conservación está en la categoría de preocupación menor (LC). Frente a ello, Flores-Ortega et al. (2015) consideran que $C$. robalito es vulnerable a la degradación de su hábitat (manglares, estuarios y zonas costeras) pese a ser una especie euritópica. Al respecto la UICN (2020) lo considera como de preocupación menor (LC). Sugerimos que la escasez podría deberse a otros factores como problemas en la distribución del pescado, es decir, una distribución no equitativa (PONTÓN, 2015).

\section{Presencia de peces pequeños}

En ocasiones por cada entrevista con los comerciantes pudimos detectar la presencia de unos pocos ejemplares juveniles en varias especies dentro de sus puestos de venta. A continuación, mencionamos las especies detectadas seguido del número de entrevistas en el que fueron registradas, así a simple vista destacaron juveniles de: $S$. sierra (2), $H$. axilaris (1), O. platypogon (1), $L$. argentiventris (1), L guttatus (1), T. obesus (2), E. lineatus (1) y especialmente de $S$. ensis (3), P. medius (4), S. oerstedii (4) y $S$. peruviana (3).

Desde nuestra perspectiva la mayoría de los peces en este estudio se veían bastante grandes y en estado de madurez sexual. Se sugiere tomar datos sobre la talla de los peces y peso de las gónadas para tener una idea más eficaz sobre su estado de desarrollo mediante el uso de la talla media de madurez sexual en caso de realizar nuevos estudios, el uso de estas medidas puede contribuir al desarrollo de una legislación que proteja el adecuado aprovechamiento de los recursos pesqueros (AUNAP y UNIMAGDALENA, 2013). Este tipo de muestreo debería hacerse cada año para determinar si existe una reducción en el tamaño de los peces, sobre todo de aquellas especies con gran volumen de pesca como $S$. ensis y P. medius (ZABALA-LEAL et al., 2018). Esto es para tener evidencias solidas de sobrepesca.

En caso de que se comprueben los aspectos mencionados anteriormente, las técnicas de pesca usadas por los pescadores, especialmente el tamaño del enmalle de las redes que se utilizan junto a el tamaño de los anzuelos, deberían ser revisadas por las autoridades para asegurarse que solo se capturen ejemplares suficientemente grandes (KELLEHER, 2008; AUNAP y UNIMAGDALENA, 2013). Muchas jurisdicciones son muy frágiles en la aplicación de medidas de control sobre las técnicas de pesca, esto puede ser por la falta de conciencia de la problemática que ello genera y la elaboración de reglamentos inadecuados (KELLEHER, 2008). Además, muchos pescadores suelen estar en contra de la aplicación de estas restricciones (KELLEHER, 2008).

\section{Alternativas usadas con los peces que no se logran vender}

El 40\% (14) de los comerciantes mencionaron que logran vender todo su pescado. El resto de los comerciantes entrevistados mencionaron algunas alternativas sobre qué hacer con los peces que no logran vender: $17 \%$ (6) de ellos bajaban los precios para venderlos más rápido; un 29\% (10) optaban por ponerlos en refrigeración durante un tiempo máximo de 3 días, luego de lo cual los tiraban; 3\% (1) mencionó que hacia harina de pescado con ellos; 9\% (3) mencionaron que regalaban sus peces a personas que se los pedían o los donaban a niños y personas de muy bajos recursos en albergues; por último un 3\% (1) mencionó que simplemente arrojan a la basura los pescados que no logran vender. Aunque se mencionó el hecho de que se arrojaban peces a la basura, no observamos este fenómeno en la realidad, no pudimos constatar este hecho y obtener evidencias materiales o fotográficas al respecto. 
Las alternativas presentadas son aparentemente eficientes desde nuestra perspectiva, sin embargo, se sugiere realizar estudios que cuantifiquen la cantidad de pescado que realmente se desperdicia. Sería incluso recomendable preguntar a los comerciantes: ¿puedes mostrarme donde arrojaste tus pescados? ¿En qué días lo haces y a qué hora?

Se sabe que los problemas de desperdicio en la pesca en Ecuador empiezan desde el descarte de las capturas marinas, que pueden ser de entre un $6 \%$ y $8 \%$; además ocurren pérdidas durante la distribución del pescado debido al deterioro durante el proceso de transporte (FAO, 2012). Se desconoce cuánto se pierde durante la distribución. Quizás una de las soluciones a este problema sea tecnificar en mayor grado las líneas de abastecimiento de pescado con camiones frigoríficos que impidan el deterioro del pescado y diversificar las industrias que generen productos en conservas y derivados del pescado (FAO, 2020). Por ejemplo, la fabricación de harinas mencionada por los comerciantes podría ser una alternativa viable para reducir el desperdicio porque el alimento tiene más probabilidades de ser vendido y no perecer al ser almacenado (FAO, 2020). Otras soluciones viables tienen que ver con la implementación de cuotas específicas para el transporte del pescado, estas no siempre son efectivas, por lo que se recomienda presentar cierta flexibilidad en este sentido (KELLEHER, 2008).

\section{CONCLUSIONES}

El presente trabajó permitió identificar algunos problemas relacionados al aprovechamiento de los recursos pesqueros con la ayuda de los comerciantes de pescado en Mercados de Quito. La presencia de ejemplares juveniles fue detectada en varias ocasiones. Entre las especies que escaseaban, una parte de los vendedores locales desconocía la causa de dicha escases, mientras que otra la atribuía a la sobrepesca. Existe cierta preferencia en el consumo por algunas especies, lo que refleja una alta explotación. De entre esas especies, Sphyraena ensis también se registró con problemas de escases. No pudimos obtener evidencias materiales de desperdicio en los mercados visitados, esto a pesar que tuvimos respuestas habladas que confirmaban este hecho. Se presentaron y revisaron algunas alternativas frente a estos problemas que son útiles. Por último, recopilar el uso de nombres comunes puede ayudará a preservar los conocimientos de los comerciantes y ser útiles en 1 identificación de especies al estar acompañados de nombres científicos.

\section{AGRADECIMIENTOS}

Los datos del presente estudio se obtuvieron mediante el consentimiento previo informado de los comerciantes de los mercados Mayorista, Chiriyacu, San Roque, Cotocollao y La Ofelia; a los cuales reiteramos nuestro agradeciendo por brindarnos un poco de su tiempo. Queremos aplaudir la generosidad y lado humano de algunos de estos comerciantes al donar sus pescados como alternativa frente al desperdicio a personas que los necesitan y albergues.

\section{REFERENCIAS BIBLIOGRÁFICAS}

ANGULO, A. Nombres comunes y técnicos de los peces de agua dulce de Costa Rica. Filología y Lingüística, v. 39, n. 2, p 77-103, 2013.

AUTORIDAD NACIONAL DE ACUICULTURA Y PESCA Y LA UNIVERSIDAD DEL MAGDALENA (AUNAP y UNIMAGDALENA). Tallas mínimas de captura para el aprovechamiento sostenible de las principales especies de peces, crustáceos y moluscos comerciales de Colombia. 1ra ed. Colombia: Convenio 058 de 2013 entre la Autoridad nacional de acuicultura y pesca y La Universidad del Magdalena, 2013. 58 p.

BOON, K. E.; HALL, S.; MCDORMAN, T.; DUNNOFF, J.; GALL, L. Overfishing of bluefin tuna: incentivizing inclusive solutions. Univ Louisv Law Rev., v. 52, p. 1-38, 2011.

BURGOS, R.; ORDÓÑEZ-DELGADO, L.; VALLE, D. Fauna acuática del Bosque Protector Colonso: indicadora de calidad del agua. 1ra ed. Ecuador: Ministerio del Ambiente del Ecuador Fundación Bosques para la Conservación y Greenchoice, 2012. 32p.

CINNER, J. E.; MCCLANAHAN, T.R. Socioeconomic factors that lead to overfishing in small-scale coral reef fisheries of Papua New Guinea. Environ Conserv., v. 33, n. 1, p. 73-80, 2006.

COLL, M.; LIBRALATO, S.; TUDELA, S.; PALOMERA, I.; PRANOVI, F. Ecosystem overfishing in the ocean. PLoS One, v. 3, n. 12, 2008.

COSTELlO, C.; GAINES, S. D.; LYNHAM, J. Can catch shares prevent fisheries collapse? Science, v. 321, p. 1678$1681,2008$.

CUCALÓN, M. CPUE, zonas de pesca y condiciones oceanográficas térmicas de los desembarques de Pampanito (Peprilus medius) en las caletas pesqueras de San Pedro y Ayangue. 2015. 106 p. Proyecto de investigación previa a la obtención del título de Biólogo Marino. Facultad de Ciencias del Mar, Universidad Estatal de la Península de Santa Elena, La Libertad, 2015. 
FAO. El estado mundial de la pesca y la acuicultura 2016. Contribución a la seguridad alimentaria y la nutrición para todos. Roma: FAO, 2016. 224 p.

FAO. Pérdidas y desperdicio de alimentos en el mundo: alcance, causas y prevención. Roma: FAO, 2012. 42 p.

FAO. Peces nativos de agua dulce de América del Sur de interés para la acuicultura: una síntesis del estado de desarrollo tecnológico de su cultivo. Roma: FAO/Serie Acuicultura en Latinoamérica, 2010. 204 p.

FAO. Perfiles de pesca y acuicultura por países: Ecuador (2011). Hojas de datos de perfiles de los países. Roma: Departamento de Pesca y Acuicultura de la FAO, 2020. Disponible en: <http://www.fao.org/fishery/facp/ECU/es.> Acceso en: 19 jun. 2020.

FAO. 2020. La pérdida y el desperdicio de alimentos en las cadenas de valor del pescado. Disponible en:

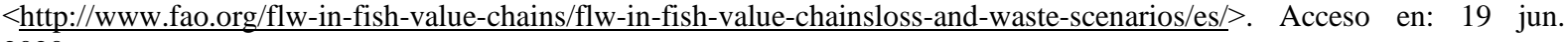
2020.

FARIÑA, Á.; RUIZ-VELÁSQUEZ, L.; ROJAS, M.; PEÑUELA, J.; GONZÁLES, N. Etnobiología marina y aspectos pesqueros en seis comunidades costeras de la Península de Paria, Venezuela. Interciencia, v. 36, n. 4, p. 256-264, 2011.

FINKBEINER, E. M. et al. Reconstructing overfishing: moving beyond Malthus for effective and equitable solutions. Fish Fish., v. 18, n. 6, p. 1180-1191, 2017

FLORES-ORTEGA, J. et al. Hábitos alimentarios de los jóvenes de Centropomus robalito (Centropomidae: Actinopterygii) en la laguna de Barra de Navidad, Jalisco, México. Revista de Biología Tropical, v. 63, n. 4, p. 1071-1081, 2015.

GARCÍA, J. R.; FIGUEROA, F. Cultura, interculturalidad, transculturalidad: elementos de y para un debate. Antropología Sociológica, v. 9, p. 15-62, 2007.

GUEVARA-CARRASCO, R. Sobrepesca de la Merluza peruana, lecciones mal entendidas. Bol. Inst. Mar Perú, v. 21, n. 1 y 2 , p. 27-32, 2004.

HOLLENSTEIN, P. ¿Están en riesgo los mercados y ferias municipales? Aprovisionamiento de alimentos, economías populares y la organización del espacio público urbano de Quito. 1ra ed. Quito-Ecuador: Friedrich-Ebert-Stiftung Ildis Ecuador, 2019. 28 p.

JIMÉNEZ-PRADO, P. et al. Guía de peces para aguas continentales en la vertiente occidental del Ecuador. $1 \mathrm{ra}$ ed. Esmeraldas-Ecuador: Pontificia Universidad Católica del Ecuador Sede Esmeraldas (PUCESE); Universidad del Azuay (UDA) y Museo Ecuatoriano de Ciencias Naturales (MECN) del Instituto Nacional de Biodiversidad, 2015. 416 p.

JIMÉNEZ-PRADO, P.; BEÁREZ, P. Peces marinos del Ecuador continental. Tomo II.: guía de especies. 1ra ed. Quito-Ecuador: SIMBIOE/NAZCA/IFEA, 2004. 406 p.

KELLEHER, K. Descartes en la pesca de captura marina mundial: una actualización. 1ra ed. Roma: FAO Documento Técnico de Pesca. No. 470. FAO, 2008. 147p.

MAIOLI-AZEVEDO, V.; DA FONSECA-KRUEL, V. S. Plantas medicinales y rituales vendidas en mercados callejeros de la ciudad de Río de Janeiro: estudio de caso de las zonas del norte y sur. Acta Bot. Brasilica, v. 21, n. 2, p. 263-275, 2007.

MARTÍN, C. Mercados municipales: la respuesta de los consumidores. Distribución y consumo, v. 109, p. 15-28, 2010.

MARTINO, P. Técnicas de pesca, percepción y manejo de la ictiofauna de la población de San Javier (Sta. Fe). Córdoba: Facultad de Ciencias Exactas, Físicas y Naturales Universidad Nacional de Córdoba, 2016. 62 p.

MOLFESE, C.; BEARE, D.; HALL-SPENCER, J. M. Overfishing and the replacement of demersal finfish by shellfish: an example from the English Channel. PLoS One, v. 9, n. 7, e101506, 2014.

NASS, P.; TORRES-SORANDO, L.; BENÍTEZ, B. Introducción al conocimiento de las pesquerías marinas en Venezuela. 1ra ed. Venezuela: Universidad Bolivariana de Venezuela, 2010. 93 p.

PARFITT, J.; BARTHEL, M.; MAGNAUGHTON, S. Desperdicio de alimentos dentro de las cadenas de suministro de alimentos: cuantificación y potencial de cambio hasta 2050. Phil. Trans. R. Soc. B., v. 365, p. 3065-3081, 2010.

PONTÓN, G. Plan de negocios para la creación de una empresa comercializadora de pesca blanca en la ciudad de Quito. 2015. 68 p. Tesis previa a la obtención del título de Ingeniero de Empresas-Facultad de Ciencias Económicas y Negocios. Universidad Tecnológica Equinoccial, Quito, 2015.

RODRÍGUEZ, Y.; VALDÉS, M.; HERNÁNDEZ, R.; SORIA, S. Guía metodológica para estudios etnobotánicos de especies forestales en comunidades amazónicas y afines. Revista cubana de ciencias forestales, v. 7, n. 1, p. 98-110, 2018.

RON, T. Estrategias para el incremento de consumo de pescado en el centro de desarrollo infantil mies. Noviembre 2011 a febrero 2012. 2012. 139 p. Disertación de grado previa a la obtención del título de licenciada en nutrición humana-Facultad de Enfermería, Pontificia Universidad Católica del Ecuador, Quito, 2012.

UICN. La lista Roja de especies amenazadas de la UICN. 2020. Disponible en: <https://www.iucnredlist.org/> Acceso en: 19 jun. 2020.

ZEBADÚA, J. Cultura, identidades y transculturalidad. Apuntes sobre la construcción identitaria de las juventudes indígenas. Revista Limina R. Estudios sociales y humanísticos, v. 9, n. 1, 2011.

ZABALA-LEAL, I. et al. Aspectos reproductivos de Sphyraena ensis (Perciformes, Sphyraenidae) habitando en la costa de San Blas Nayarit, sudeste del Golfo de California. California Fish and Game, v. 104, n. 1, p. 7-18, 2018.

ZHOU, S.; SMITH, A. D.; KNUDSEN, E. E. Ending overfishing while catching more fish. Fish and Fisheries, v. 16, n. 4, p. 716-722, 2015. 